\title{
Entropic Inhibition: How the Activity of a AAA+ Machine Is Modulated by Its Substrate-Binding Domain
}

\author{
Marija Iljina,* Hisham Mazal, Pierre Goloubinoff, Inbal Riven, and Gilad Haran* \\ Cite This: ACS Chem. Biol. 2021, 16, 775-785 \\ Read Online
}

ABSTRACT: $\mathrm{ClpB}$ is a tightly regulated AAA+ disaggregation machine. Each ClpB molecule is composed of a flexibly attached N-terminal domain (NTD), an essential middle domain (MD) that activates the machine by tilting, and two nucleotide-binding domains. The NTD is not well-characterized structurally and is commonly considered to serve as a dispensable substrate-binding domain. Here, we use single-molecule FRET spectroscopy to directly monitor the real-time dynamics of ClpB's NTD and reveal its unexpected autoinhibitory function. We find that the NTD fluctuates on the microsecond

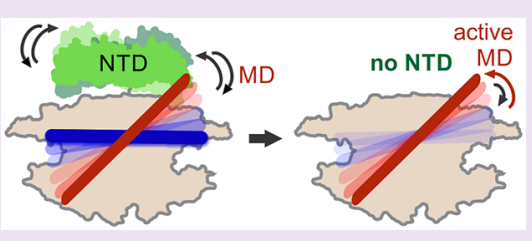
time scale, and these dynamics result in steric hindrance that limits the conformational space of the MD to restrict its tilting. This leads to significantly inhibited ATPase and disaggregation activities of $\mathrm{ClpB}$, an effect that is alleviated upon binding of a substrate protein or the cochaperone DnaK. This entropic inhibition mechanism, which is mediated by ultrafast motions of the NTD and is not dependent on any strong interactions, might be common in related ATP-dependent proteases and other multidomain proteins to ensure their fast and reversible activation.

$\mathrm{N}$ -terminal domains (NTDs) tune the functions of numerous proteins by autoinhibitory intramolecular interactions with cognate domains ${ }^{1}$ and by propagating allosteric signals to remote sites through disordered linkers. Examples include control of gating in the neurotransmitter receptor NMDA upon binding of allosteric drugs to the NTD, ${ }^{3}$ activation of the regulation of the unfoldase $\mathrm{ClpC}$ upon binding of its NTD to the adaptor protein $\mathrm{MecA},{ }^{4}$ control of proteolytic activity of the $20 \mathrm{~S}$ core-particle proteasome by fast conformational switching of NTD residues, ${ }^{5}$ or suppression of the protease ClpP due to the unfolding of its NTD. ${ }^{6}$ NTDbearing $\mathrm{AAA}+$ proteins (ATPases associated with diverse cellular activities) are large ATP-dependent molecular machines ${ }^{7}$ with a diverse set of tightly regulated functions. ${ }^{8}$ The bacterial heat-shock protein $\mathrm{ClpB}$ is an AAA+ machine that works in collaboration with the DnaK chaperone system (DnaK, DnaJ, and GrpE) to rescue proteins from aggregates and is essential for conferring thermotolerance., ${ }^{9,10}$ In its functional form, $\mathrm{ClpB}$ is a ring-shaped homohexamer that disaggregates substrate proteins by actively threading them through its central channel in an ATP-dependent process. ${ }^{11}$ Each monomer of $\mathrm{ClpB}$ comprises several domains ${ }^{12}$ (Figure 1a): a flexibly connected NTD, two ATP-binding domains (NBD1 and NBD2), and the regulatory middle domain (MD) that activates the machine by ultrafast toggling. ${ }^{13}$ The NTD is a globular, 135-147-residue $\alpha$-helical domain, with a structure that is highly conserved across the $\mathrm{ClpA}, \mathrm{ClpB}$, and $\mathrm{ClpC}$ subfamilies $^{14}$ and in eukaryotic homologues. ${ }^{15}$ In bacteria, a truncated variant of $\mathrm{ClpB}$ lacking the $\mathrm{NTD}, \Delta \mathrm{NClpB}$, is naturally coexpressed as a minor product together with the fulllength protein ${ }^{16,17}$ and was reported to contribute significantly to bacterial thermotolerance. ${ }^{18}$ The biological relevance of the a

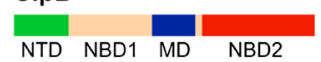

b

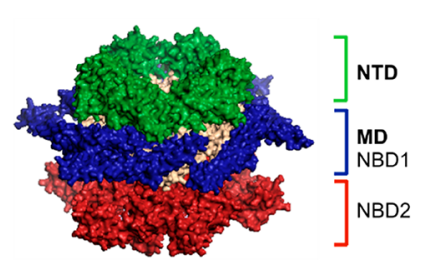

$\triangle \mathrm{NCIpB}$

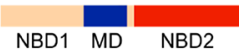

c

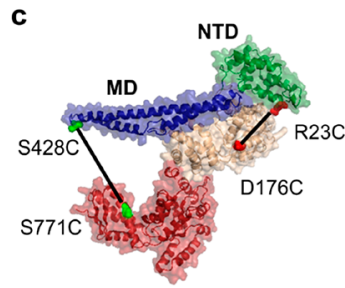

Figure 1. Structure of $\mathrm{ClpB}$. (a) The sequence of domains in the fulllength ClpB. A truncated, naturally occurring variant of $\mathrm{ClpB}$, $\triangle \mathrm{NClpB}$, lacks the NTD. (b) Structure of the ClpB hexamer with the NTDs highlighted in green (PDB: 1QVR). ${ }^{12,30}$ (c) Structure of the $\mathrm{ClpB}$ monomer with the NTD shown in green and the MD in blue. Pairs of residues mutated to cysteines for the incorporation of fluorescent dyes are highlighted: R23C and D176C (in red) for the characterization of NTD dynamics; S428C and S771 (in green) for the measurement of MD dynamics.

truncated variant, compared to the full-length protein, is not clear.

Received: March 4, 2021

Accepted: March 10, 2021

Published: March 19, 2021 
a

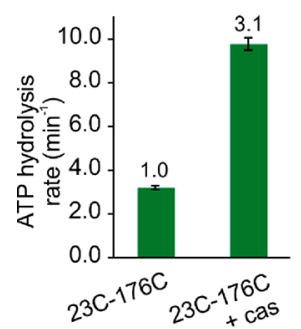

b

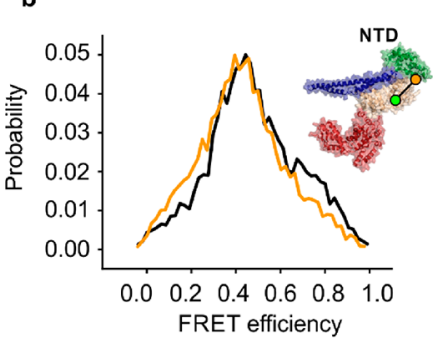

c

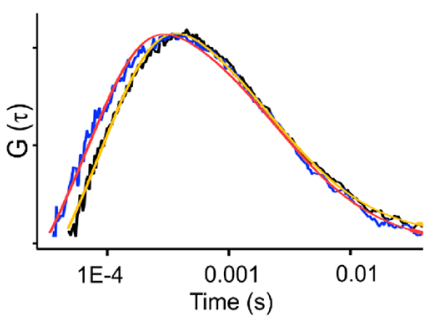

Figure 2. Analysis of the conformational dynamics of the NTD. (a) ATPase activity of fully labeled 23C-176C protein, either basal or in the presence of $25 \mu \mathrm{M} \kappa$-casein $(n=3)$. The error bars correspond to standard errors of the mean. The numbers above the bars designate relative activity. (b) FRET efficiency histograms of the 23C-176C mutant of ClpB with a single labeled protomer in a $2 \mathrm{mM}$ ATP solution, either in the absence of substrate-protein (black) or in the presence of $25 \mu \mathrm{M} \kappa$-casein (yellow). Here and elsewhere, FRET efficiency histograms are areanormalized. (c) Filtered FCS cross-correlation curves of $23 \mathrm{C}-176 \mathrm{C} \mathrm{ClpB}$ without (black) and with $25 \mu \mathrm{M} \kappa$-casein (blue). The red and yellow curves are fits to eq 1 in the Methods and yield similar diffusion coefficients but faster dynamics in the presence of $\kappa$-casein (red) than in its absence (yellow) - see text.

The six NTDs of ClpB form a distinct large ring at the top of the hexamer (Figure 1b). ${ }^{12}$ These domains serve as initial binding sites for certain substrate-proteins ${ }^{19,20}$ prior to their processing. Because of its high mobility, the entire NTD ring is frequently unobserved in cryo-electron microscopy (cryo-EM) reconstructions of $\mathrm{ClpB}$ hexamers. ${ }^{21-24}$ However, two recent cryo-EM studies employed "focused classification" to capture several substrate-bound NTDs. ${ }^{25,26}$ They revealed NTD trimers that made contacts with substrate protein molecules and surrounded the central channel of ClpB. In addition, a full ring of six NTDs was resolved in a cryo-EM structure of the yeast homologue of $\mathrm{ClpB}, \mathrm{Hsp} 104$, also showing that they interact with the substrate. ${ }^{27}$ The substrate-binding properties of the NTDs were also characterized in several biochemical studies, ${ }^{19,28,29}$ and the substrate-binding groove was mapped by NMR spectroscopy. ${ }^{20}$

Although the experimentally validated function of the NTDs in $\mathrm{ClpB}$ remains limited to substrate-protein engagement, accumulating evidence suggests that their role might be more complex. Several studies reported an increased ATP hydrolysis rate by the NTD-truncated mutant $\triangle \mathrm{NClpB}$ in the absence of protein substrates, compared to the full-length $\mathrm{ClpB}$, indicating that the NTDs may inhibit futile ATP hydrolysis through yet uncharacterized communication pathways. ${ }^{17,19,20,31-36}$ Indeed, potentially relevant interactions of the NTD with the MD and NBDs in solution were recently detected by X-ray footprinting in Hsp 104. ${ }^{37}$ Furthermore, the function of the NTDs during substrate processing and translocation remains uncertain. Recently, an NTD deletion was reported to abolish the translocation of maltose-binding protein. ${ }^{38}$ On the other hand, $\Delta \mathrm{NClpB}$ remained active in multiple in vitro disaggregation assays in the presence of DnaK-DnaJ-GrpE with other preaggregated protein substrates, implying a secondary role for the NTD in this process. ${ }^{20,28,29,32,39}$ It was proposed that the NTDs block the central channel ${ }^{20}$ and can undergo conformational changes to actively assist with the translocation and disaggregation of substrate-proteins. ${ }^{29} \mathrm{NMR}$ spectroscopy in aqueous solution suggested fast movement of the NTDs. ${ }^{20}$ These dynamics have not been studied in detail and the time scales of the motion, any accompanying conformational rearrangements, and their implications for the machine function remain to be characterized.

To shed light on the equivocal roles of the NTDs in ClpB, we have used a combination of single-molecule FRET (smFRET) spectroscopy and biochemical experiments. We directly monitor the submillisecond motions of the NTDs and demonstrate that engagement of substrate proteins does not restrict these conformational dynamics. We characterize the communication of the NTD with other functional domains of $\mathrm{ClpB}$ and find that the ultrafast motions of the NTD restrict sterically the MD conformations, thus maintaining the inactive state of the MD. This mechanism, which we term entropic inhibition, results in suppressed ATPase and disaggregation activities of $\mathrm{ClpB}$. Our results suggest that the NTD is critically involved in the reversible autoinhibition of $\mathrm{ClpB}$ through its ultrafast dynamics.

\section{RESULTS}

The NTD Fluctuates on the Microsecond Time Scale with Changes in Conformational Dynamics upon Substrate Binding. In the crystal structure of the full-length Thermus thermophilus (TT) ClpB (henceforth ClpB), ${ }^{12}$ the NTDs are connected to the neighboring NBD1 domains by disordered linkers, which might suggest that these domains are highly mobile. Analysis of the NTD dynamics in a related AAA + hexameric machine p97 by solution NMR spectroscopy suggested microsecond motions. ${ }^{40}$ Here, we set to characterize the real-time dynamics of the NTD within the ClpB hexamer in aqueous solution. As in our recent study of the $\mathrm{MD}$ dynamics in the full-length $\mathrm{ClpB},{ }^{13}$ we employed a combination of smFRET and a maximum likelihood analysis method $\left(\mathrm{H}^{2} \mathrm{MM}\right)$, described in detail previously. ${ }^{41}$ Briefly, $\mathrm{H}^{2} \mathrm{MM}$ is a hidden Markov model technique that analyzes smFRET data on the level of individual photons in order to extract fast dynamics. To study the NTD dynamics, we located a cysteine for fluorescent labeling on the NTD, at the end of $\alpha$ helix A1 (residue R23), and a second cysteine was inserted into a rigid position within NBD1 (residue D176) of ClpB (Figure 1c). The resulting double mutant of $\mathrm{ClpB}$ was labeled with Alexa Fluor 488 (AF488) and Alexa Fluor 594 (AF594; Methods). It was confirmed that the fluorescently labeled 23C176C construct exhibited unaltered ATPase activity $(3.2 \pm 0.1$ $\min ^{-1}$ relative to $3.5 \pm 0.2 \mathrm{~min}^{-1}$ in WT ClpB).

The labeled protein showed prominent ATPase stimulation upon binding of the model protein substrate $\kappa$-casein ${ }^{42}$ (3.1fold enhancement, Figure $2 \mathrm{a}$ ). We have shown before that ClpB hexamers are stable and demonstrate no assembly defects under our experimental conditions, using cryo-EM and interprotomer smFRET experiments. ${ }^{13}$ Here, we used native gel electrophoresis to demonstrate that the $23 \mathrm{C}-176 \mathrm{C}$ 

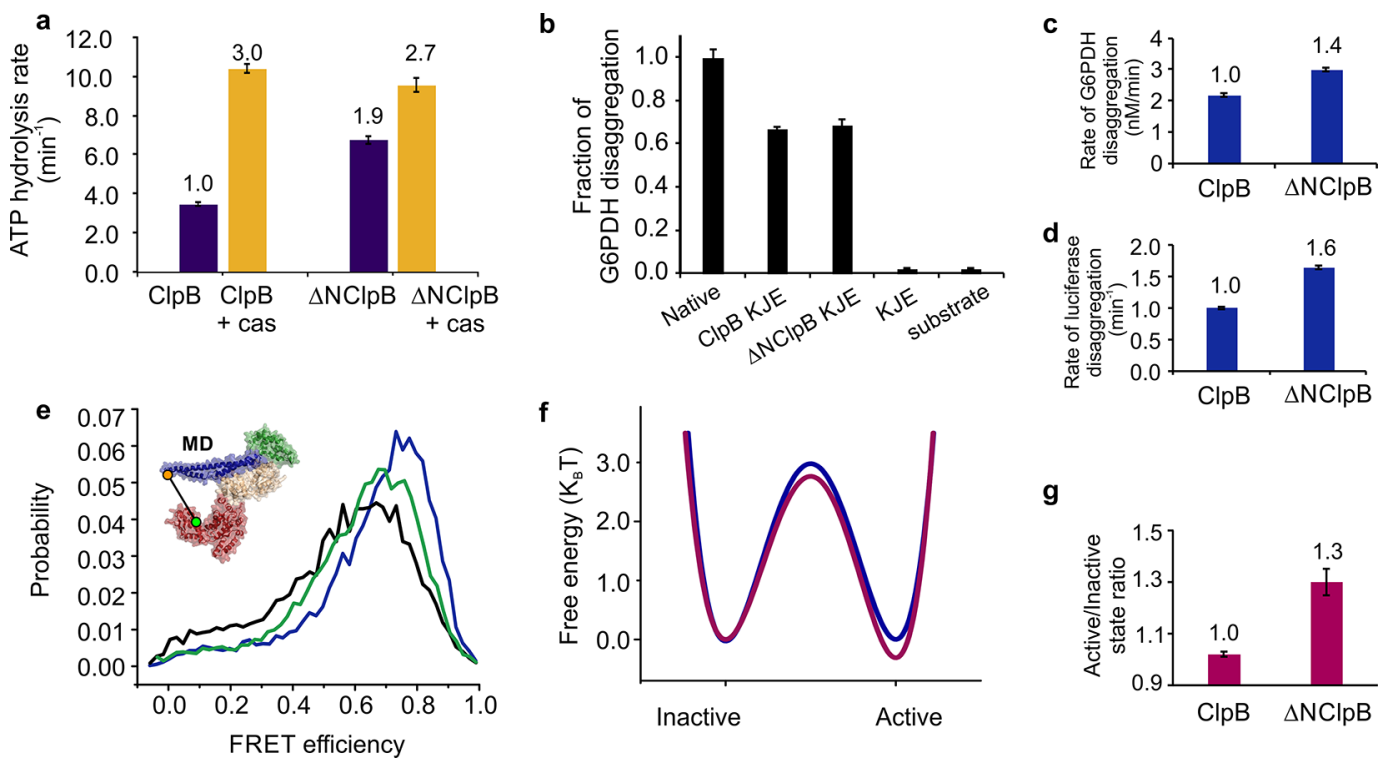

Figure 3. ATPase, disaggregation activities, and MD dynamics of $\mathrm{ClpB}$ and $\Delta \mathrm{NClpB}$. (a) Rate of ATP hydrolysis measured at $25{ }^{\circ} \mathrm{C}$ with and without $50 \mu \mathrm{M} \kappa$-casein ("cas," $n=3$ ). (b) Disaggregation of heat-induced aggregates of G6PDH as a substrate, measured after $3 \mathrm{~h}$ of incubation with ClpB variants $(2 \mu \mathrm{M})$ in the presence of DnaK, DnaJ, and GrpE $(2 \mu \mathrm{M}, 1 \mu \mathrm{M}$, and $1 \mu \mathrm{M}$, respectively). The fraction of disaggregation was determined by taking the activity of native (nonaggregated) G6PDH as 1 (labeled "Native", $n=6)$. (c,d) Rate of disaggregation of heat-induced aggregates of G6PDH (c) or of firefly luciferase (d; see disaggregation yields in the main text) by ClpB variants in the presence of DnaK, DnaJ, and GrpE $(n=3)$. (e) FRET efficiency histograms of the full-length S428C-S771C construct of ClpB (black) and of the respective $\Delta$ NClpB mutant in the presence of $2 \mathrm{mM}$ ATP (green) and in the presence of $25 \mu \mathrm{M} \kappa$-casein (blue). (f) Comparison of the free-energy profiles of MD dynamics of the full-length $\mathrm{ClpB}$ (blue) and $\Delta \mathrm{NClpB}$ (purple), calculated from $\mathrm{H}^{2} \mathrm{MM}$ parameters using the Arrhenius equation with a pre-exponential factor of $10^{5} \mathrm{~s}^{-1}$. (g) The population ratio of horizontal (inactive) to tilted (active) states of the MD, derived from $\mathrm{H}^{2} \mathrm{MM}$ analysis. The error bars in all panels correspond to standard errors of the mean. In a, c, d, and e, values above the bars are the fold increases relative to the corresponding values for $\mathrm{ClpB}$ (which are set to 1 ).

construct had no assembly defects in the presence of $2 \mathrm{mM}$ ATP (Figure S1). Furthermore, fluorescence anisotropy measurements of single-cysteine mutants (23C and 176C) indicated that the dyes attached at these positions showed unrestricted rotation that was not affected by the presence of $\kappa$ casein (Figure S1).

Double-labeled ClpB subunits were mixed with a 100 -fold molar excess of unmodified unlabeled $\mathrm{ClpB}$ subunits (WT $\mathrm{ClpB}$ ), ensuring that the newly assembled ClpB hexamers contained only one fluorescently labeled protomer. We conducted photon-by-photon smFRET measurements on diffusing molecules of the construct introduced above in the presence of $2 \mathrm{mM}$ ATP. The arrival times of the photons emitted by both donor (AF488) and acceptor (AF594) dyes were registered on two separate detectors. After selecting computationally molecules that contained both AF488 and AF594 dyes (Methods and Figure S4), we calculated FRET efficiency histograms. The histograms were broad (Figure $2 b$ ), indicating multiple conformations of the NTD. Surprisingly, there was a significant shift to lower FRET efficiency values in the presence of $\kappa$-casein, meaning that longer distances between residues $23 \mathrm{C}$ and $176 \mathrm{C}$ became more populated. This indicates that the NTD's equilibrium positions become closer to the central channel, in agreement with previous proposals that the NTDs direct bound substrate-proteins toward the central pore. ${ }^{29}$ We made sure that the shift of the FRET efficiency histograms was not due to photophysical effects by comparing properties of fluorescence bursts in experiments with and without $\kappa$-casein (Table S1). In a control experiment, we relocated one dye from the NTD to NBD2 (739C), and the FRET efficiency histograms of the mutant
176C-739C were identical with and without $\kappa$-casein (Figure $\mathrm{S} 1$ ), supporting the lack of unwanted photophysical effects.

To assess the time scale of the NTD motion within ClpB hexamers, we analyzed the dynamics by fluorescence lifetime correlation spectroscopy (FLCS). This experimental technique $^{43}$ enables filtering fluorescence cross-correlation functions to obtain the contribution of separate subspecies observed in the FRET histogram (see Methods). We calculated crosscorrelation functions between the range of species in the histograms of Figure $2 b$ with the lowest FRET efficiency values $(<0.18)$ and the range of species with the highest FRET efficiency values $(>0.75)$, to obtain the overall exchange rate between these population ranges. The resulting curves (Figure $2 c)$ showed a prominent rise of the signal on the microsecond time scale, and analysis indicated conformational dynamics with an exchange rate of $8330 \pm 630 \mathrm{~s}^{-1}$. Interestingly, the addition of $\kappa$-casein at a concentration of $25 \mu \mathrm{M}$ led to acceleration of the dynamics to $12500 \pm 780 \mathrm{~s}^{-1}$. The reasons for the observed increase in the NTD dynamics with $\kappa$-casein elude us at this point in time (see Discussion).

For a more quantitative analysis of the various states occupied by the NTD, we applied $\mathrm{H}^{2} \mathrm{MM}$ to individual smFRET photon trajectories, ${ }^{41}$ using a model with three states, which was the smallest number of states required to properly fit the smFRET data (see below). From this analysis, we could learn about the states in the system and the rates of their interconversion. The $\mathrm{H}^{2} \mathrm{MM}$-derived FRET efficiency values were $0.18,0.49$, and 0.81 for states 1,2 and 3 , correspondingly, and the transition rates out of these states were $3540 \pm 480$ $\mathrm{s}^{-1}, 1860 \pm 150 \mathrm{~s}^{-1}$, and $2500 \pm 220 \mathrm{~s}^{-1}$, respectively, and were increased in the presence of $\kappa$-casein (Figure S1, Table 
S2), consistent with the FLCS analysis. We compared the $\mathrm{H}^{2} \mathrm{MM}$-derived FRET efficiency states to the theoretical values based on available ClpB structures (Supplementary Methods and Table S3). From this analysis, state 1 at a FRET efficiency of 0.18 was consistent with the NTD conformation captured in the cryo-EM structure of substrate-unbound $\mathrm{ClpB}$ where this domain plugs the central channel, ${ }^{25}$ and state 3 at 0.81 was in agreement with the NTD conformation found in the crystal structure of $\mathrm{ClpB} .^{12}$

To validate these results, we performed an analysis of dwell time distributions, ${ }^{13,44}$ and the resulting escape rates were in close agreement with the values obtained from $\mathrm{H}^{2} \mathrm{MM}$ (Figure S1, Table S2), suggesting that three states fit the data well. From the $\mathrm{H}^{2} \mathrm{MM}$-derived FRET efficiency values of states 1 and 3 (0.18 and 0.81), we calculated the amplitude of the NTD motion to be $\sim 28 \AA$. Together, these results show that, within the hexamer, the NTD undergoes ultrafast large-scale movements that are independent of nucleotide state and are only mildly accelerated by the binding of the model substrateprotein. The high speed and conformational freedom are likely to underlie all of the NTD-driven regulations in ClpB that we characterize in subsequent sections.

The NTD Suppresses ATPase and Disaggregation Activities of ClpB. To further understand the role of the NTD in ClpB's activity, we expressed a truncated version, $\Delta \mathrm{NClpB}$, which lacks the first 140 residues of the protein (Figure S3). We confirmed by native polyacrylamide gel electrophoresis and by size-exclusion chromatography that both $\mathrm{ClpB}$ and $\triangle \mathrm{NClpB}$ were well-assembled (Figure S2). Measurements of ATP hydrolysis rates in the presence of 2 $\mathrm{mM}$ ATP at $25{ }^{\circ} \mathrm{C}$ yielded a doubled value for $\Delta \mathrm{NClpB}$ compared to $\mathrm{ClpB}\left(6.8 \pm 0.3 \mathrm{~min}^{-1}\right.$ vs $3.5 \pm 0.2 \mathrm{~min}^{-1}$; Figure $3 \mathrm{a})$, in close agreement with previous literature values. ${ }^{13,32,45}$

However, in the presence of $\kappa$-casein $(50 \mu \mathrm{M})$, similar maximal hydrolysis rates were reached by $\mathrm{ClpB}$ and $\Delta \mathrm{NClpB}$ $\left(10.4 \pm 0.4 \mathrm{~min}^{-1}\right.$ and $9.6 \pm 0.6 \mathrm{~min}^{-1}$, respectively, Figure $3 a)$. The results suggest that the truncated $\mathrm{ClpB}$ variant is dysregulated and exhibits futile ATPase activity in the absence of protein substrate. We compared the disaggregation activity of $\mathrm{ClpB}$ and $\Delta \mathrm{NClpB}$ by monitoring the reactivation of heatinduced aggregates of glucose-6-phosphate dehydrogenase $(\mathrm{G} 6 \mathrm{PDH})^{46}$ and observed a $\sim 67 \%$ regeneration of soluble G6PDH activity by both ClpB and $\Delta \mathrm{NClpB}$ (Figure $3 \mathrm{~b}$ ). Even though the yields of disaggregation by the two variants were essentially the same, $\Delta \mathrm{NClpB}$ displayed a higher disaggregation rate compared to the full-length variant $(3.0 \pm 0.1 \mathrm{nM}$ $\min ^{-1}$ vs $2.2 \pm 0.1 \mathrm{nM} \mathrm{m^{-1 }}{ }^{-1}$ Figure $\left.3 \mathrm{c}\right)$. This difference in the rates of disaggregation was also observed in experiments with heat-induced aggregates of firefly luciferase $\left(21.7 \pm 0.5 \mathrm{~min}^{-1}\right.$ for $\Delta \mathrm{NClpB}$ compared to $13.1 \pm 0.65 \mathrm{~min}^{-1}$ for $\mathrm{ClpB}$; Figure $3 \mathrm{~d}$ ), in which the final yields of luciferase regeneration were 43 $\pm 3 \%$ for $\mathrm{ClpB}$ and $76 \pm 5 \%$ for $\Delta \mathrm{NClpB}$.

smFRET Experiments Reveal That $\triangle$ NClpB Has Activated MDs. To shed light on the effect of the NTD deletion on $\mathrm{ClpB}$ activity, we probed the deletion influence on MD dynamics by using smFRET measurements and $\mathrm{H}^{2} \mathrm{MM}$ analysis. $^{13,41}$ In a recent study, we reported that the MDs of $\mathrm{ClpB}$ are highly dynamic, moving on the submillisecond time scale between their collinear (inactive) and tilted (active) states. $^{13}$ Our results indicated that the MD is a continuous (analog), rather than a two-state (digital), activation switch for $\mathrm{ClpB}$, with the population ratio of its states affecting the overall activity of the machine. Strikingly, in the absence of substrates or cochaperones, the two states in the full-length $\mathrm{ClpB}$ were populated equally, with an active/inactive population ratio of $1.00 \pm 0.01$, and were rapidly interconverting with transition rates of $k_{12}=5300 \pm 150 \mathrm{~s}^{-1}$ and $k_{21}=5700 \pm 100 \mathrm{~s}^{-1}$, respectively. This population ratio was shown to be significantly modulated by a range of allosteric signals, such as the binding of DnaK, nucleotides, and substrate proteins.

To characterize the MD dynamics of $\Delta$ NClpB by smFRET, we generated a double-cysteine mutant of $\Delta \mathrm{NClpB}$ (Figure 1c). The first cysteine was introduced into motif 1 of the MD (residue 288, which corresponds to 428 in the full-length $\mathrm{ClpB}$ ) and the second into NBD2 (residue 631, corresponding to 771 in the full-length ClpB, Figure 1c). The resulting double mutant of $\triangle \mathrm{NClpB}$ was labeled with AF488 and AF594 (Methods) and mixed with a 100-fold molar excess of unmodified $\Delta \mathrm{NClpB}$ to obtain a single fluorescently labeled protomer within each hexamer. We tested the effect of labeling on $\Delta \mathrm{NClpB}$, and found no change in ATPase or disaggregation activity (Figure $\mathrm{S} 2$ ), in agreement with previous findings on the full-length $\mathrm{ClpB} .{ }^{13}$ smFRET measurements were carried out on $\Delta \mathrm{NClpB}$ in solution, in the presence of 2 $\mathrm{mM}$ ATP. The addition of ATP guaranteed the stability of ClpB hexamers in all our smFRET experiments; ${ }^{13}$ indeed, FRET efficiency histograms showed no evidence for their dissociation during the measurements (Figure S5).

FRET efficiency histograms of $\triangle \mathrm{NClpB}$ appeared to be shifted to higher average FRET efficiency values compared to the data for the full-length $\mathrm{ClpB}$ (Figure 3e). The two major states of the MD were found to possess FRET efficiency values of $0.8 \pm 0.01$ (state 1 ) and $0.48 \pm 0.01$ (state 2), in good agreement with the active and inactive states of the full-length variant. ${ }^{13}$ In contrast to the results for the full-length $\mathrm{ClpB}$, though, the states were unequally populated, with relative populations of $0.56 \pm 0.02$ and $0.44 \pm 0.02$, respectively, and an increased active/inactive state ratio of $1.30 \pm 0.05$ instead of $1.00 \pm 0.01$ (Figure $3 \mathrm{~g}$ ), commensurate with the overall shift of the FRET histogram to higher value. As in the fulllength $\mathrm{ClpB}$, the two states were found to be under fast exchange, with interconversion rates of $k_{12}=4800 \pm 200 \mathrm{~s}^{-1}$ (from active to inactive state) and $k_{21}=6300 \pm 90 \mathrm{~s}^{-1}$ (reverse direction; Table S5). Approximate free energy profiles of the $\mathrm{MD}$ dynamics, constructed based on the derived rates, showed that the energy barrier for the transition from inactive to active state was decreased in $\triangle \mathrm{NClpB}$, and the active state was stabilized (Figure 3f). These results indicate that the $\mathrm{MD}$ in the $\Delta \mathrm{NClpB}$ variant toggles between the same conformations as in the full-length variant but, remarkably, favors the active conformation. On the basis of our previous findings, the higher active/inactive state ratio should lead to a larger disaggregation rate of $\Delta \mathrm{NClpB},{ }^{13}$ as is indeed observed (Figure 3c,d). To find whether this activation of the $\mathrm{MD}$ in $\triangle \mathrm{NClpB}$ could be enhanced further by protein substrates, we repeated the experiments in the presence of $25 \mu \mathrm{M} \kappa$-casein. The resulting FRET efficiency histograms showed higher average FRET efficiency values (Figure 3e, Tables S4 and S5) due to a further increase in the active/inactive state ratio to $1.76 \pm 0.01$, indicating that additional activation of the MD in $\triangle \mathrm{NClpB}$ can take place in response to substrate binding.

The NTD Suppresses the MD through Direct Contacts. Next, we set out to explain why the MD appears activated upon the deletion of the NTD. It was previously found biochemically that the isolated NTD of ClpB from Escherichia coli (E. coli) does not form stable contacts with 
a

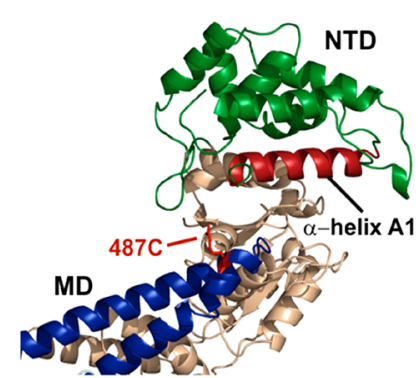

C

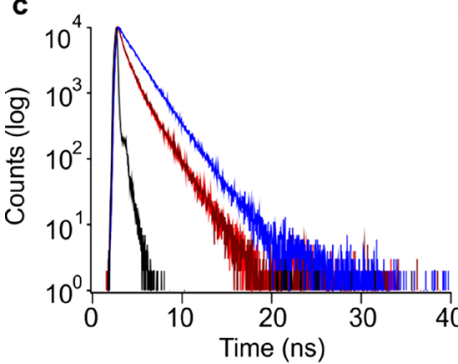

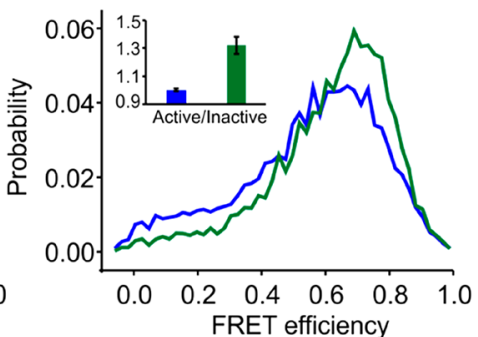

b

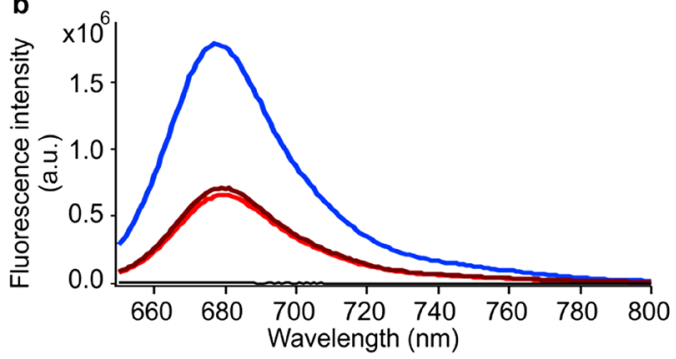

e

Figure 4. NTD mutants of ClpB affect MD dynamics. (a) Zoom into the upper region of ClpB protomer (crystal structure PDB: 1 QVR). ${ }^{12}$ The MD is colored in blue, and the NTD in green, with $\alpha$-helix A1 of the NTD highlighted in red. Residue 487C on the MD used for Atto 655 incorporation is shown as red sticks. (b) Steady-state fluorescence spectra of Atto 655-labeled ClpB mutants: ClpB (blue), 12W-ClpB (red), 23W$\mathrm{ClpB}$ (dark red), buffer (black). Average fractions of fluorescence intensity, normalized to the result of the mutant without incorporated tryptophan, were 0.36 for $12 \mathrm{~W}$ and 0.39 for $23 \mathrm{~W}$. (c) Fluorescence decay curves: ClpB (blue), 12W-ClpB (red), 23W-ClpB (dark-red), instrument response function (black). The resulting fluorescence lifetimes were $2 \mathrm{~ns}$ for the control ClpB mutant and $1.3 \mathrm{~ns}$ for $12 \mathrm{~W}$ and $23 \mathrm{~W}$ variants. (d) FRET efficiency histograms of the full-length $\mathrm{ClpB}$ (blue) and the truncated $\Delta \mathrm{HA} 1 \mathrm{ClpB}$ variant (green). Inset shows the corresponding active/ inactive state ratios of the MD. (e) ATPase activity of $\Delta \mathrm{HAlClpB}$, compared to the full-length and $\Delta \mathrm{NClpB}$ variants $(n=4)$. (f) Rate of disaggregation of G6PDH aggregates in the presence of DnaK, DnaJ, and GrpE ( $2 \mu \mathrm{M}, 1 \mu \mathrm{M}$, and $1 \mu \mathrm{M}$, respectively, $n=3)$. The error bars correspond to standard errors of the mean.

other parts of the molecule, ${ }^{47}$ and we confirmed this result for $T T \mathrm{ClpB}$, used in this study. $\triangle \mathrm{NClpB}$ and the isolated NTD, comprising residues $1-141$ of $\mathrm{ClpB}$, that were mixed together (at $40 \mu \mathrm{M} \Delta \mathrm{NClpB}$ and $650 \mu \mathrm{M}$ NTD) migrated separately in size exclusion chromatography and native polyacrylamide gel electrophoresis analysis (Figure S6), indicating that no stable complex was formed.

Since the NTD is connected to the neighboring NBD1 by a flexible linker region, ${ }^{12}$ it is interesting to ask whether its rapid motions in aqueous solution allow it to reach the MD. We first qualitatively assessed this possibility by using a simple computational procedure that sampled the conformational space of the NTD (see Methods section). From the inspection of the crystal structure of $\mathrm{ClpB},{ }^{12}$ we found that the region of the MD that is closest to the NTD is the edge of its motif 2 . Starting with the crystal structure of the ClpB monomer, ${ }^{12}$ we generated multiple conformations of the NTD within the context of the full hexamer by fully rotating it around a single residue in the flexible linker region (position 142). Then, we selected NTD residues that were located within $10 \AA$ from the edge of motif 2 (residue 487) in the generated conformations (Figure 4a) and found 11 in total, five of which were on or close to $\alpha$-helix A1 of the NTD (these residues are indicated in Figure S3). On the basis of this finding, we designed ClpB mutants in order to test whether we can experimentally detect an interaction between $\alpha$-helix A1 and the MD. To this end, we used Atto 655, a fluorophore that is effectively quenched upon the interaction with several amino acids, especially with tryptophan (W), leading to a significant decrease in fluorescence signal. ${ }^{48} \mathrm{We}$ introduced a cysteine into the MD (position 487) of the full-length $\mathrm{ClpB}$ for labeling with maleimide-linked Atto 655. We then incorporated single tryptophan residues into the NTD, either at position 12 or 23 within $\alpha$-helix A1 (Figure S3), and used a mutant without these additional tryptophans as a control. Fully labeled ClpB mutants Atto 655-487C-ClpB, Atto 655-487C-12W-ClpB, and Atto $655-487 \mathrm{C}-23 \mathrm{~W}-\mathrm{ClpB}$ were found to be wellassembled in the presence of $2 \mathrm{mM}$ ATP and showed ATPase activity that was comparable to that of unmodified ClpB. 487C-ClpB variants were fully active in disaggregation experiments before labeling but had no disaggregation activity when fully labeled, likely due to the hindrance of the DnaK binding site ${ }^{49}$ on the MD by Atto 655 (Figure S7).

Bulk steady-state fluorescence measurements of the Atto 655-labeled variants (Figure 4b) showed a strong decrease (around 60\%) of the fluorescence intensity in the tryptophancontaining mutants relative to the control $487 \mathrm{C}-\mathrm{ClpB}$, and the fluorescence lifetimes of these variants were found to be shortened (from $2 \mathrm{~ns}$ in the control samples to $1.3 \mathrm{~ns}$ in $12 \mathrm{~W}$ and $23 \mathrm{~W}$-containing variants). These differences indicate quenching of the fluorophore by the tryptophan residues in the NTD. Since the fractional decrease in fluorescence intensity of the mutants was larger than the decrease in fluorescence lifetime, we concluded that the quenching process has both a static component, due to the formation of a nonfluorescent Atto 655-quencher complex, and a dynamic component, due to transient encounters of the fluorophore with the quencher. ${ }^{50}$ This finding is commensurate with previous results on the effect of tryptophan on Atto 655 fluorescence. ${ }^{48}$ Considering that the dye is located on the tip of 

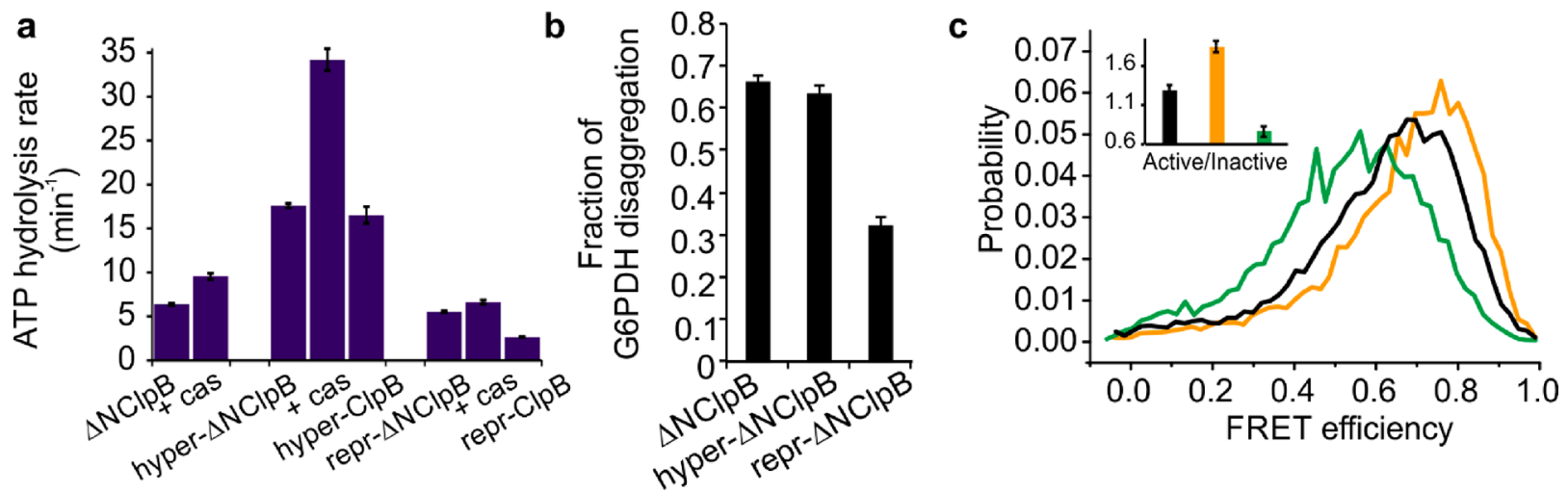

Figure 5. Characterization of MD-NBDs allosteric pathways in $\triangle \mathrm{NClpB}$. (a) The rate of ATP hydrolysis of activated and repressed $\Delta \mathrm{NClpB}$ mutants at $25^{\circ} \mathrm{C}$ with and without $(n=3-5) 25 \mu \mathrm{M} \kappa$-casein. The basal rates of full-length hyper- and repr-ClpB are included for reference. ${ }^{13}$ (b) Disaggregation yield of heat-induced aggregates of G6PDH by $\triangle \mathrm{NClpB}$ mutants, measured after $3 \mathrm{~h}$ of disaggregation in the presence of DnaK, DnaJ, and GrpE $(2 \mu \mathrm{M}, 1 \mu \mathrm{M}$, and $1 \mu \mathrm{M}$, respectively, $n=2)$. (c) FRET efficiency histograms of activated and repressed mutants of $\Delta N C l p B$ : $\Delta \mathrm{NClpB}$ (black), hyper- $\Delta \mathrm{NClpB}$ (orange), repr- $\Delta \mathrm{NClpB}$ (green). Inset shows the corresponding active/inactive state ratios of the MD. The error bars correspond to standard errors of the mean.

the $\mathrm{MD}$, these results are in agreement with our hypothesis that the NTD can make contacts with the MD.

In order to determine whether the contacts that the NTD makes with the MD are sufficient to affect the MD dynamics, we removed the entire $\alpha$-helix A1 from the NTD by deleting residues $8-25$ of the full-length $\mathrm{ClpB}$ (Figure 4a, Figure S3). This truncated mutant $(\triangle \mathrm{HA} 1 \mathrm{ClpB})$ was fully assembled (Figure S7), and smFRET analysis of its double-labeled variant revealed that its $\mathrm{MD}$ was activated in comparison with the MD of the full-length ClpB. Indeed, FRET efficiency histograms (Figure 4d) were shifted to higher FRET values when compared with the histograms for the full-length ClpB. The derived interconversion rates of the $\mathrm{MD}$ were unequal, $k_{12}=$ $4400 \pm 100 \mathrm{~s}^{-1}$ and $k_{21}=5900 \pm 300 \mathrm{~s}^{-1}$ (Table S5), resulting in an increased active/inactive state ratio of $1.32 \pm 0.06$, comparable to the ratio found in $\Delta \mathrm{NClpB}$ (Table S4). Measurements of the ATP hydrolysis rate of $\Delta \mathrm{HA1ClpB}$ yielded a value of $4.6 \pm 0.1 \mathrm{~min}^{-1}$ (Figure $4 \mathrm{e}$ ), intermediate between the activity of the full-length $\mathrm{ClpB}\left(3.5 \mathrm{~min}^{-1}\right)$ and $\Delta \mathrm{NClpB}\left(6.8 \mathrm{~min}^{-1}\right)$. The rate of disaggregation of heattreated $\mathrm{G} 6 \mathrm{PDH}$ by $\triangle \mathrm{HA} 1 \mathrm{ClpB}$ was higher than by the fulllength $\mathrm{ClpB}\left(3.2 \pm 0.2 \mathrm{nM} \min ^{-1}\right.$ vs $2.2 \pm 0.1 \mathrm{nM} \mathrm{min}{ }^{-1}$, Figure 4f). Therefore, removal of the interactions with $\alpha$-helix A1 of the NTD activates the MD. This is consistent with the fluorescence quenching results, which indicate a direct contact of $\alpha$-helix A1 with the MD. These results imply that $\alpha$-helix A1 comprises residues that can reach the $\mathrm{MD}$ and significantly affect its conformational transitions.

NTD Removal Does Not Disrupt Communication between the MD and NBDs. Several mutations that are located remotely from the NTD or the NTD-NBD1 linker have been shown to promote or repress the activity of ClpB. ${ }^{22,51,52}$ We asked whether these mutations still have the same effect in $\mathrm{ClpB}$, after its NTD is deleted. The MD is known to be tightly regulated through a network of salt bridges that connect it to the neighboring NBD1. ${ }^{2,51,52}$ To check whether this regulatory pathway is retained upon the NTD deletion, we generated activated and repressed hexamers of $\Delta \mathrm{NClpB}$ by introducing previously characterized single mutations that were expected to alter the MD dynamics (Figure S3). Point-mutation E209A in NBD1 (termed hyper$\Delta \mathrm{NClpB}$, corresponding to E349A in the full-length $\mathrm{ClpB}$ ) breaks a salt bridge connecting it to NBD1, which results in a detached and activated MD. ${ }^{52}$ The hyper- $\Delta$ NClpB variant showed 3.5-fold higher rate of ATP hydrolysis than the unmodified $\triangle \mathrm{NClpB}$, an increased disaggregation rate $(3.3 \pm$ $\left.0.1 \mathrm{nM} \mathrm{min}{ }^{-1}\right)$, and the same disaggregation yield as $\Delta \mathrm{NClpB}$ (Figure 5a,b). These results are consistent with the reported effects of similar mutations in the full-length $\mathrm{ClpB}^{13}$ and in $\Delta$ NClpB from E. coli. ${ }^{53}$ The FRET efficiency histogram of the hyper- $\Delta \mathrm{NClpB}$ variant was shifted to higher values compared to $\Delta \mathrm{NClpB}$ data (Figure $5 \mathrm{c}$ ). The transition rates of the $\mathrm{MD}$, extracted from the analysis of smFRET trajectories of this mutant, were altered (Table S5), $k_{12}=4100 \pm 400 \mathrm{~s}^{-1}$ and $k_{21}$ $=7800 \pm 400 \mathrm{~s}^{-1}$, resulting in the relative populations of the active and inactive states of $0.65 \pm 0.01$ and $0.35 \pm 0.01$. Consequently, the active/inactive state ratio was $1.85 \pm 0.07$ (Table S4), higher than in $\Delta \mathrm{NClpB}$, and similar to the result for the activated mutant of the full-length protein. ${ }^{13}$

Point-mutation E283A within the MD (repr- $\Delta \mathrm{NClpB}$, corresponding to E423A in the full-length $\mathrm{ClpB}$ ) stabilizes its contacts with NBD1 and with MDs of neighboring subunits. $^{22,51,54,55}$ Repr- $\Delta$ NClpB had a slightly decreased (by $10 \%)$ ATPase activity compared to $\Delta \mathrm{NClpB}$. Its disaggregation yield was reduced by half compared to the nonmutated $\Delta \mathrm{NClpB}$ (Figure 5a,b), and the rate of disaggregation was significantly lowered $\left(0.05 \pm 0.1\right.$ nM.min $\left.{ }^{-1}\right)$, in good agreement with the previously observed effects of this mutation in the full-length protein. ${ }^{13,54,55}$ The FRET efficiency histogram of repr- $\Delta \mathrm{NClpB}$ was shifted to lower average values in comparison to $\triangle \mathrm{NClpB}$. The derived interconversion rates were $k_{12}=5500 \pm 150 \mathrm{~s}^{-1}$ and $k_{21}=4200 \pm 300 \mathrm{~s}^{-1}$ (Table S5), leading to an increased relative population of the inactive state $(0.57 \pm 0.04)$ and the active/inactive state ratio of $0.76 \pm$ 0.07 , in agreement with the result of this mutation in the fulllength ClpB (Table S4). ${ }^{13}$ Therefore, both the activating and repressing mutations strongly affected the MD dynamics of $\Delta \mathrm{NClpB}$. This suggests that the salt bridges between the MD and NBD1 that regulate the $\mathrm{MD}$ conformations in $\mathrm{ClpB}$ remain unaffected by NTD deletion.

We also probed another previously characterized pathway of $\mathrm{MD}$ regulation that involves the nucleotide binding sites of ClpB. Recently, we found that ATP binding to NBD1 decreased the population of the active state of the $M D$, 

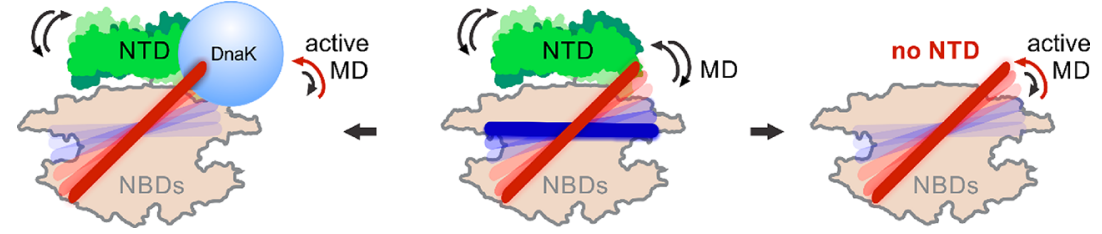

Figure 6. Schematic summary of entropic inhibition in ClpB by its NTD. Center: In the full-length ClpB, the NTD (green) and the MD undergo microsecond-time-scale motions (shown by reversible arrows). The MD is in dynamic equilibrium between active/inactive states (red/dark blue) with a state population ratio of $\sim 1$. The NTD limits the conformational space of the MD by exerting a steric exclusion effect through its ultrafast movements. This in turn leads to autoinhibition of ATPase and disaggregation activities of ClpB but does not preclude potential DnaK binding to the MD. Left: DnaK binding to the MD (light blue, not drawn to scale) leads to a higher population of the active state of the MD, which in turn activates $\mathrm{ClpB}$, as we reported in our previous study. ${ }^{13}$ Right: In the $\triangle \mathrm{NClpB}$ variant, which lacks the NTD, the MD suppression by the NTD is lost, and it is free to sample its full conformational space. This also results in a more populated active state of the MD, with an increase of the active/inactive state ratio to $\sim 1.3$, and an overall activation of $\mathrm{ClpB}$ even in the absence of DnaK. In bacterial cells, $>20 \%$ of the molecules of ClpB lack the NTD domain and may contribute around a third of the overall thermotolerance, ${ }^{18}$ making this variant of physiological importance.

whereas ATP binding to NBD2 had the opposite effect. ${ }^{13} \mathrm{We}$ set to determine whether this regulation was retained in $\Delta \mathrm{NClpB}$. To this end, we generated and analyzed mutants of the Walker A motifs, which are located in the two NBDs and are responsible for ATP binding (Figure S8). ${ }^{56}$ We found no difference in their activity or in their MD dynamics in comparison to the corresponding full-length $\mathrm{ClpB}$ mutants, ${ }^{13}$ suggesting that NTD removal does not affect the allosteric regulation of the $\mathrm{MD}$ conformations upon nucleotide binding.

\section{DISCUSSION}

In this work, we studied the dynamics of the NTD and its communication with other domains of $\mathrm{ClpB}$ using a powerful combination of smFRET experiments, photon-by-photon $\mathrm{H}^{2} \mathrm{MM}$ analysis, fluorescence quenching measurements, and biochemical assays. We found that the NTD is involved in a novel mechanism of autoinhibition. This finding is unexpected: while the regulatory role of the MD was firmly established by multiple previous studies to act as the main regulatory switch of ClpB's activity, ${ }^{13,22,49,51,57}$ the NTD is often viewed as a nonessential substrate-docking domain. ${ }^{32}$ Indeed, through its microsecond-time-scale dynamics, the NTD is able to exert an internal volume exclusion effect that significantly restricts the conformational space of the regulatory $\mathrm{MD}$ of $\mathrm{ClpB}$ and suppresses its tilting. This new mode of inhibition through conformational fluctuations, which we term entropic inhibition, directly depends on the fast motions of the NTD in and out of the conformational space of the $\mathrm{MD}$, does not require strong interactions, and ultimately leads to inhibited bulk activities of ClpB. More specifically, our fluorescence quenching data showed that $\alpha$-helix A1 of the NTD makes steric contacts with motif 2 of the MD. The apparent restriction of the $\mathrm{MD}$ motions by the NTD results in the suppression of ATPase activity and the inhibition of the disaggregation by $\mathrm{ClpB}$ (Figure 6). We directly verified the importance of the steric effect of the NTD on the MD by observing that NTD removal resulted in tilting of the $\mathrm{MD}$ in $\mathrm{ClpB}$, shifting its active/ inactive population ratio from 1 to 1.3 , which in turn activated the machine and increased both its ATPase and disaggregation activities.

The entropic inhibition of ClpB by its NTD involves the functionally important motif 2 of the MD. This motif comprises the DnaK-binding epitope of $\mathrm{ClpB}^{49}$ essential to its disaggregation function..$^{22,49,57}$ Importantly, the fast movements of the NTD facilitate the approach of a DnaK molecule toward the $\mathrm{MD}$ and its binding, ${ }^{49}$ which removes the inhibition and can cause the release of bound substrate- proteins. ${ }^{58}$ However, as long as a DnaK molecule has not bound to the MD, the overall activity level of the machine remains low. This yet unrecognized role of the NTD as a regulatory element for ClpB's inactivation is depicted in Figure 6. The NTD operates through its physical interaction with the major regulatory $\mathrm{MD}$ and prevents ClpB's futile activity in the absence of substrate-protein and DnaK binding.

Previous studies have established the substrate-binding properties of the NTDs. ${ }^{19,20,28,29,59}$ It was found that the NTD is crucial for the binding of large protein aggregates that would otherwise not be recognized by $\mathrm{ClpB}^{28}$ and that the NTD of Hsp104 could facilitate dissolution of prions, which was not possible in the Hsp104 lacking the NTD. ${ }^{59}$ Therefore, the NTD has a dual function. In the basal state, in the absence of substrate-protein binding, the NTD serves to limit the ATPase and the disaggregation activities of the machine by the entropic inhibition mechanism. Upon binding of specific aggregated protein substrates by the NTDs, the inhibition is released and thus the machine activity is initiated.

Notably, our data suggest that the NTDs slightly reorient themselves with respect to NBD1 upon substrate-protein binding becoming closer to the central channel. This behavior is to be contrasted with p97, mentioned above, whose NTDs undergo a large upward movement with out-of-plane rotation of $75^{\circ}$ upon activation. ${ }^{60}$ The presence of these conformational changes in the NTD upon substrate-protein binding is in agreement with recent cryo-EM structure of $\mathrm{ClpB}$, where a trimer of substrate-bound NTDs from alternating protomers was shown to form a ring at the channel entrance, maintaining the overall helical arrangement of the protomers. ${ }^{26}$ Nevertheless, the NTD actually moves faster by $\sim 50 \%$ upon substrate binding, which might have an effect on the dynamics of DnaK binding to the $\mathrm{MD}$, thereby influencing the activation state of the whole machine. To note, an AAA+ protein ClpA, which is structurally very similar to $\mathrm{ClpB}$, has an almost identical NTD ${ }^{14}$ but no MDs. The addition by evolution of an $\mathrm{MD}$ in $\mathrm{ClpB}$ may have allowed the incorporation of an additional layer of control that involves autoinhibition by the NTD and activation by DnaK binding.

Consistent with the autoinhibitory role of the NTD, $\triangle \mathrm{NClpB}$ is an activated $\mathrm{ClpB}$ variant that has tilted MDs and elevated basal ATP hydrolysis rate, and shows high disaggregation activity toward heat-induced aggregates of G6PDH and firefly luciferase. The increased basal ATP activity exhibited by $\Delta \mathrm{NClpB}$ in comparison to the full-length variant is in good agreement with multiple preceding studies $^{17,19,20,31-36}$ and implies that this truncated mutant of 
$\mathrm{ClpB}$ is more wasteful in terms of ATP consumption in the absence of bound substrate-proteins. Our data show unaltered yield and a faster rate of disaggregation following the NTD removal. Thus, we can conclude that $\Delta \mathrm{NClpB}$ is a more active disaggregase, at least toward certain protein substrates. The presence of a small fraction of the $\Delta \mathrm{NClpB}$ variant, with its high intrinsic ATPase activity and increased disaggregation activity, might be crucial for cell survival under conditions of particularly harsh heat shock and stress.

We conclude that the NTD is involved both in substrate binding and in the crucial autoinhibition of machine activation through the mechanism of entropic inhibition. Thus, the conformational fluctuations of the NTD prevent the allosteric switch of $\mathrm{ClpB}$, the $\mathrm{MD}$, from activating the machine. The NTD is absent in a significant fraction of naturally synthesized bacterial ClpB molecules, which lack this autoinhibition. Even more importantly, the fast motions of the NTD do not preclude the binding of DnaK to the $\mathrm{MD}$, which also rescues it from the partially inhibited state, generating a fully active machine. A similar multimodal allosteric control through flexibly connected and fast-moving domains is likely to be present in numerous $\mathrm{AAA}+$ proteins and other multidomain molecular machines to enable their rapid regulation under changing cellular conditions.

\section{METHODS}

Protein Expression and Purification. Thermus thermophilus $\mathrm{ClpB}$ (TT. ClpB); its $\triangle \mathrm{NClpB}$ variant beginning at residue 141 (Val); its mutants (Figure S3), cochaperones DnaK, DnaJ, and GrpE, cloned into a pET28b vector; and the isolated $\mathrm{N}$-terminal domain of $\mathrm{ClpB}$ (residues 1-141), cloned into pProEx vector, were expressed and purified according to recently published protocols, ${ }^{13}$ as detailed in the Supplementary Methods.

ATP Activity Measurements. The ATP activity of ClpB variants was measured using a coupled colorimetic assay. ${ }^{61} \mathrm{ClpB}$ or its mutants ( $1 \mu \mathrm{M}$ total monomer concentration) were incubated with 2 $\mathrm{mM}$ ATP and an ATP regeneration system (2.5 mM phosphoenol pyruvate, 10 units $/ \mathrm{ml}$ pyruvate kinase, 15 units $/ \mathrm{ml}$ lactate dehydrogenase, $2 \mathrm{mM}$ 1,4-dithioerythritol, $2 \mathrm{mM}$ EDTA, $0.25 \mathrm{mM}$ $\mathrm{NADH}$ ) in $50 \mathrm{mM}$ HEPES ( $\mathrm{pH} 8), 50 \mathrm{mM} \mathrm{KCl}$, and $0.01 \%$ Tween 20 . For the experiments in the presence of the model substrate $\kappa$ casein (Sigma-Aldrich), it was added to a final concentration of 50 $\mu \mathrm{M}$. ATP hydrolysis was initiated by the addition of $\mathrm{MgCl}_{2}(10 \mathrm{mM})$ and measured by monitoring the time-dependent decrease in $\mathrm{NADH}$ absorption at $340 \mathrm{~nm}$ at $25^{\circ} \mathrm{C}$. Data were background-corrected in all cases, and the measured ATP hydrolysis rate per $\mathrm{ClpB}$ monomer per minute is presented.

Disaggregation of Heat-Induced Aggregates of G6PDH and Luciferase. Disaggregation experiments were performed following previously described procedures. ${ }^{13,46,62}$ The protocol for aggregate preparation and disaggregation reactions is detailed in the Supplementary Methods.

Labeling of ClpB and $\Delta \mathrm{NClpB}$ Variants. Labeling of 23C-176C mutant of ClpB, S288C-S631C $\Delta \mathrm{NClpB}$, and any of its mutational variants was performed similarly to the previously reported protocol. ${ }^{13}$ First, the double-cysteine mutant of $\mathrm{ClpB}$ (or $\Delta \mathrm{NClpB}$ ) was incubated for $1 \mathrm{~h}$ with a 1:1.2 molar ratio of Alexa Fluor (AF) 594 (C5 maleimide, Invitrogen) under native conditions (25 mM HEPES, $25 \mathrm{mM} \mathrm{KCl}, \mathrm{pH} 7)$. The unreacted dye was removed on a desalting column (Sephadex G25, GE Healthcare). The protein was exchanged into buffer containing guanidinium chloride in order to fully expose unlabeled cysteine residues (25 mM HEPES, $25 \mathrm{mM} \mathrm{KCl,} 2 \mathrm{M}$ $\mathrm{GdmCl}, \mathrm{pH} 7$ ) and incubated with a 1:1.5 molar ratio of AF488 (C5 maleimide, Invitrogen) for $1 \mathrm{~h}$ to achieve complete labeling of unreacted cysteines, followed by the separation of unreacted dye on a desalting column. Labeling was confirmed by absorption measurements, and the fraction of the double-labeled AF488/594 species was consistently $>30 \%$ as directly calculated from single-molecule measurements. Labeling of the full-length $487 \mathrm{C}-\mathrm{ClpB}$ variants with Atto 655-maleimide (Sigma-Aldrich) was carried out by incubating $\mathrm{ClpB}$ with the dye at a 1:1.5 ratio for $5 \mathrm{~h}$ in the dark (25 mM HEPES, $25 \mathrm{mM} \mathrm{KCl}, 2 \mathrm{M} \mathrm{GdmCl}, \mathrm{pH} 7$ ), followed by the separation of unreacted dye on a desalting column (Sephadex G25, GE Healthcare), buffer-exchange to the native buffer ( $25 \mathrm{mM}$ HEPES, $25 \mathrm{mM}$ $\mathrm{KCl}, \mathrm{pH} 7$ ), and filtration of the labeled protein through a $0.22 \mu \mathrm{m}$ filter (Millex, Millipore).

Mixing of Fluorescently Labeled and Unlabeled Protomers. For the preparation of $\mathrm{ClpB}$ and $\triangle \mathrm{NClpB}$ assemblies for smFRET experiments, AF488-AF594-labeled double-cysteine mutants were combined with a 100 -fold molar excess of unlabeled cysteine-less $\mathrm{ClpB}$ or $\Delta \mathrm{NClpB}$, respectively. This ratio ensured that the probability of the incorporation of one labeled protomer in a hexamer was $5.7 \%$, whereas the probability to find two labeled protomers in the same hexamer was as low as $0.15 \%$. To achieve full mixing, the protein solutions were initially dialyzed in the presence of $6 \mathrm{M} \mathrm{GdmCl}$. This was followed by dialysis steps in the presence of $4,2,1$, and $0 \mathrm{M}$ $\mathrm{GdmCl}$. The final steps involved extensive dialysis into a low-salt buffer (25 mM HEPES, $25 \mathrm{mM} \mathrm{KCl}, 10 \mathrm{mM} \mathrm{MgCl} 2,2 \mathrm{mM} \mathrm{ATP,} \mathrm{pH}$ 8 ) and filtration through $0.1 \mu \mathrm{m}$ filters (Whatman Anotop-10). The assembled $\Delta \mathrm{NClpB}$ was aliquoted, flash-frozen, and stored at $-80^{\circ} \mathrm{C}$ until further use. For the preparation of activated, repressed $\Delta \mathrm{NClpB}$ and its Walker A mutant hexamers, fluorescently double-labeled $\Delta \mathrm{NClpB}$ bearing the respective mutation was mixed with the 100 -fold excess of unlabeled $\Delta \mathrm{NClpB}$ with the same mutation but without cysteine residues, and dialyzed according to the above protocol. For the preparation of $\triangle \mathrm{HAlClpB}$ hexamers of $\mathrm{ClpB}$, double-labeled $\Delta \mathrm{HA} 1 \mathrm{ClpB}$ was mixed with the $\triangle \mathrm{HA} 1 \mathrm{ClpB}$ mutant of $\mathrm{ClpB}$ without cysteine residues. These labeling and mixing procedures result in correctly folded protein, as shown in our previous study, ${ }^{13}$ and as can be inferred from the ATPase and disaggregation activities, which are similar to those of the wild-type protein.

Single-Molecule Measurements. The preparation of custommade glass flow chambers for single-molecule experiments was carried out following previously reported protocols ${ }^{41,44,63}$ and is detailed in the Supplementary Methods. The chambers were coated with a supported lipid bilayer composed of egg phosphatidylcholine (Avanti Polar Lipids) to prevent protein absorption during measurements. The assembled hexamers of $\mathrm{ClpB}$ (or $\Delta \mathrm{NClpB}$ ) were diluted to $\sim 50$ $\mathrm{pM}$ of labeled $\mathrm{ClpB}$, corresponding to $\sim 5 \mathrm{nM}$ of total $\mathrm{ClpB}$, into a single-molecule buffer ( $25 \mathrm{mM}$ HEPES, $25 \mathrm{mM} \mathrm{KCl}, 10 \mathrm{mM} \mathrm{MgCl}$, 2 mM ATP, $0.01 \%$ Tween 20, pH 8). The solutions were loaded into the chambers, which were rapidly sealed to prevent evaporation. Measurements were conducted using a home-built inverted confocal single-molecule microscope,${ }^{41,44,63}$ which is described in detail in the Supplementary Methods. Data collection was carried out on freely diffusing molecules as reported. ${ }^{13}$ Briefly, the samples were illuminated with focused and overlapped 485 and $594 \mathrm{~nm}$ diode laser beams pulsed at a ratio of 3:1 with a repetition rate of $40 \mathrm{MHz}$. The emitted photons were divided into two channels using a dichroic mirror (FF580-FDi01, Semrock) and passed through band-pass filters, ET-535/70m for the AF488 emission and ET-645/75m for the AF594 emission (Chroma). The arrival times of the emitted photons in both channels were registered by single-photon avalanche photodiodes (PerkinElmer SPCM-AQR-15) coupled to a standalone time-correlated single photon counting module (HydraHarp 400, PicoQuant). Data were acquired for up to $3.5 \mathrm{~h}$ per sample at ambient temperature $\left(22^{\circ} \mathrm{C}\right)$. Between two and four individual samples were prepared and measured for each $\mathrm{ClpB}$ variant and condition.

smFRET Data Analysis. Data analysis was performed as recently described. ${ }^{13}$ A cutoff of $10 \mu \mathrm{s}$ was used to effectively separate fluorescence bursts from the background. FRET efficiency and stoichiometry were calculated as detailed elsewhere. ${ }^{64}$ We applied several corrections to eliminate any artifacts and to ensure that only fluorescent photons arising from individual molecules entered the analysis. More details on the data selection can be found in the Supporting Information section, Figure S4. FRET efficiency values were corrected for the leakage of photons from the donor to acceptor 
channel (estimated to be no more than 7\%). Using stoichiometry/ FRET efficiency histograms (Figure S4), any singly labeled species were eliminated, and only the molecules containing both AF488 and AF594 were selected for further analysis. For the photon-by-photon $\mathrm{H}^{2} \mathrm{MM}$ analysis, fully detailed previously, ${ }^{41}$ the photons arising from preselected molecules after donor-only excitation were used. A fixed number of molecules (5800) was analyzed for each sample. The data were fitted with a three-state model. In the case of the analysis of MD dynamics, relative populations of the two major states were extracted and compared between different $\Delta \mathrm{NClpB}$ mutants or conditions (Table S4). This fitting approach was rigorously tested and confirmed through multiple additional methods on the equivalent full-length $\mathrm{ClpB}$ data sets in our preceding study. ${ }^{13}$

FLCS Measurements. Conventional smFRET measurements of freely diffusing double-labeled molecules of $23 \mathrm{C}-176 \mathrm{C}$ hexamers (assembled in 1:100 ratio with WT ClpB) were performed. Pulsed interleaved excitation by 485 and $594 \mathrm{~nm}$ lasers was used (in 1:1 sequence). The measurements were conducted with laser powers of $50 \mu \mathrm{W}(485 \mathrm{~nm})$ and $10 \mu \mathrm{W}(594 \mathrm{~nm})$, a repetition rate of $40 \mathrm{MHz}$ and a TCSPC time resolution of 16 ps. Fluorescence lifetime components were extracted from the low FRET efficiency (below 0.18 ) and the high FRET efficiency (above 0.75 ) populations. Photon weights were calculated for the two lifetime components (low FRET and high FRET population), according to Kapusta et al. ${ }^{43}$ Subsequently, the same ClpB samples were measured at $1 \mathrm{nM}$, with pulsed excitation by the $485 \mathrm{~nm}$ laser at $50 \mu \mathrm{W}, 40 \mathrm{MHz}$, and a TCSPC time resolution of 16 ps. Filtered FCS cross-correlation functions were calculated using the photon weights of the low FRET and high FRET populations as previously described. ${ }^{43}$ The filtered cross-correlation curves were fitted to the equation (Origin Pro 2019):

$$
C(t)=1+\frac{1}{N}\left(1+\frac{t}{T_{\text {dif }}}\right)^{-1}\left(1+\frac{t}{R^{2} T_{\text {dif }}}\right)^{-0.5}\left(1-A \mathrm{e}^{-t / t_{\mathrm{c}}}\right)
$$

where $N$ is the number of molecules in the confocal volume, $T_{\text {dif }}$ is the diffusion time, $R$ is the ratio of the dimensions of the Gaussian-shaped beam waist parallel and perpendicular to the direction of light propagation, $A$ is an amplitude, and $t_{c}$ is the correlation time. The value of $R$ was kept constant (at 6.2) for the fitting and was determined from the FCS calibration measurement using a reference Rhodamine 6G solution with a manufacturer-provided diffusion coefficient $\left(3.84 \times 10^{-6} \mathrm{~cm}^{2} \mathrm{~s}^{-1}\right.$ in water at $\left.22.4{ }^{\circ} \mathrm{C}\right)$. The results are shown in Figure 2c.

Rotational Simulation of the NTD. To obtain further information on the possible interactions of the NTD with the MD, we simulated multiple conformations of the NTD around its linker and mapped out possible interactions with the MD. First, we used the structural model of $T T \mathrm{ClpB}^{12}$ to create multiple conformations of the NTD by rotating it as a rigid body around a point within the NTDNBD1 linker (residue 142). We then excluded all conformations that caused a steric clash within the same protomer or with adjacent protomers. In our case, a clash was defined as a van der Waals (VDW) contact between the $\mathrm{MD}$ atoms and the other atoms of the $\mathrm{ClpB}$ hexameric complex (eq 2):

$$
0 \leq d_{i j}<R_{v}(i)+R_{v}(j)-0.3
$$

where $R_{v}(i)$ and $R_{v}(j)$ are the VDW radii of atoms $i$ and $j$, respectively, and $d_{i j}$ is the distance between the two atoms. In eq 2, $0.3 \AA$ is our clash threshold value (lower bound for a VDW contact), which was calculated from the structure of the hexameric model. ${ }^{30}$ We excluded all conformations that had at least one distance within the ranges calculated using eq 2 . We then calculated the distances between all the NTD residues and residue 487 on the MD. A threshold of $10 \AA$ was used to select NTD structures within this range of distances. We found that $\alpha$-helix A1 of the NTD was in close proximity to the MD (Figure S3).

Fluorescence Measurements of Atto 655-Labeled ClpB. Steady-state fluorescence of Atto 655 labeled ClpB mutants (either with no additional tryptophans (Ws), $12 \mathrm{~W}$ or $23 \mathrm{~W}$ ) was measured using a Fluorolog-3 spectrofluorometer (Horiba Jobin Yvon, Edison, NJ, USA). The excitation wavelength was set to $635 \mathrm{~nm}$, and fluorescence was collected between 650 and $800 \mathrm{~nm}$. Measurements were conducted using $0.5 \mu \mathrm{M}$ solutions of Atto 655-labeled $\mathrm{ClpB}$ mutants in $25 \mathrm{mM}$ HEPES, $25 \mathrm{mM} \mathrm{KCl}, 10 \mathrm{mM} \mathrm{MgCl}_{2}$, and $2 \mathrm{mM}$ ATP, at $\mathrm{pH} 8$ and at ambient temperature $\left(25^{\circ} \mathrm{C}\right)$. All spectra were collected in duplicate per mutant and background-corrected. Integrated average fluorescence values are listed in Table S6.

Ensemble Fluorescence Lifetime Measurements. Fluorescence lifetime measurements were performed on a FluoroHub timecorrelated single-photon counting instrument (Jobin-Yvon), by using a time-correlated single-photon counting method. ${ }^{48} 50 \mathrm{nM}$ solutions of Atto $655-\mathrm{ClpB}$ mutants, prefiltered through $0.1 \mu \mathrm{m}$ filters (Whatman Anotop-10), were measured (in $25 \mathrm{mM}$ HEPES, 25 $\mathrm{mM} \mathrm{KCl}, 10 \mathrm{mM} \mathrm{MgCl}, 2 \mathrm{mM} \mathrm{ATP}, \mathrm{pH} 8$ ), with four repeats per sample. A pulsed diode laser with an emission at $635 \mathrm{~nm}$ was used as an excitation source, with a pulse length of 360 ps (FWHM). The emission was collected at $680 \mathrm{~nm}$, with a slit width of $12 \mathrm{~nm}$. Photons were collected in 2048 channels, with a peak maximum of 10000 counts. Data were globally fitted with a two-exponential model using a built-in fitting wizard, and average fluorescence lifetimes are reported (Table S7).

\section{ASSOCIATED CONTENT}

\section{(S) Supporting Information}

The Supporting Information is available free of charge at https://pubs.acs.org/doi/10.1021/acschembio.1c00156.

Figures S1-S8; Tables S1-S7; Supplementary Methods (PDF)

\section{AUTHOR INFORMATION}

\section{Corresponding Authors}

Marija Iljina - Department of Chemical and Biological Physics, Weizmann Institute of Science, Rehovot 761001, Israel; @ orcid.org/0000-0002-0824-3707;

Email: Marija.Iljina@weizmann.ac.il

Gilad Haran - Department of Chemical and Biological Physics, Weizmann Institute of Science, Rehovot 761001, Israel; $\odot$ orcid.org/0000-0003-1837-9779;

Email: Gilad.Haran@weizmann.ac.il

\section{Authors}

Hisham Mazal - Department of Chemical and Biological Physics, Weizmann Institute of Science, Rehovot 761001, Israel; (1) orcid.org/0000-0002-2071-9552

Pierre Goloubinoff - Department of Plant Molecular Biology, Faculty of Biology and Medicine, University of Lausanne, CH-1015 Lausanne, Switzerland

Inbal Riven - Department of Chemical and Biological Physics, Weizmann Institute of Science, Rehovot 761001, Israel

Complete contact information is available at: https://pubs.acs.org/10.1021/acschembio.1c00156

\section{Author Contributions}

M.I., H.M., I.R., and G.H. designed the research. M.I., H.M., and P.G. performed research. M.I., H.M., and P.G. analyzed data. M.I., H.M., I.R., P.G., and G.H. wrote the paper.

\section{Notes}

The authors declare no competing financial interest.

\section{ACKNOWLEDGMENTS}

M.I. is the recipient of an EMBO Long-Term Fellowship (ALTF 317-2018). H.M. was supported by Planning \& 
Budgeting Committee of the Council of Higher Education of Israel. P.G. is funded by the Swiss National Science Foundation (Grant 31003A_156948). G.H. is funded by the European Research Council (ERC) under the European Union's Horizon 2020 research and innovation programme (grant agreement No 742637) and is the incumbent of the Hilda Pomeraniec Memorial Professorial Chair. We are grateful to Y. Barak for experimental support. We thank R. Rosenzweig for $\mathrm{ClpB}$ plasmids and for excellent advice.

\section{REFERENCES}

(1) Pufall, M. A., and Graves, B. J. (2002) Autoinhibitory domains: modular effectors of cellular regulation. Annu. Rev. Cell Dev. Biol. 18, 421-462.

(2) Ma, B., Tsai, C. J., Haliloğlu, T., and Nussinov, R. (2011) Dynamic allostery: linkers are not merely flexible. Structure 19 (7), 907-917.

(3) Gielen, M., Retchless, B. S., Mony, L., Johnson, J. W., and Paoletti, P. (2009) Mechanism of differential control of NMDA receptor activity by NR2 subunits. Nature 459 (7247), 703-707.

(4) Wang, F., Mei, Z., Qi, Y., Yan, C., Hu, Q., Wang, J., and Shi, Y. (2011) Structure and mechanism of the hexameric MecA-ClpC molecular machine. Nature 471 (7338), 331-335.

(5) Religa, T. L., Sprangers, R., and Kay, L. E. (2010) Dynamic regulation of archaeal proteasome gate opening as studied by TROSY NMR. Science 328 (5974), 98-102.

(6) Vahidi, S., Ripstein, Z. A., Bonomi, M., Yuwen, T., Mabanglo, M. F., Juravsky, J. B., Rizzolo, K., Velyvis, A., Houry, W. A., Vendruscolo, M., Rubinstein, J. L., and Kay, L. E. (2018) Reversible inhibition of the ClpP protease via an $\mathrm{N}$-terminal conformational switch. Proc. Natl. Acad. Sci. U. S. A. 115 (28), E6447-E6456.

(7) Hanson, P. I., and Whiteheart, S. W. (2005) AAA+ proteins: have engine, will work. Nat. Rev. Mol. Cell Biol. 6 (7), 519-529.

(8) Ogura, T., and Wilkinson, A. J. (2001) AAA+ superfamily ATPases: common structure-diverse function. Genes Cells 6 (7), 575597.

(9) Glover, J. R., and Lindquist, S. (1998) Hsp104, Hsp70, and Hsp40: a novel chaperone system that rescues previously aggregated proteins. Cell 94 (1), 73-82.

(10) Goloubinoff, P., Mogk, A., Zvi, A. P., Tomoyasu, T., and Bukau, B. (1999) Sequential mechanism of solubilization and refolding of stable protein aggregates by a bichaperone network. Proc. Natl. Acad. Sci. U. S. A. 96 (24), 13732-13737.

(11) Weibezahn, J., Tessarz, P., Schlieker, C., Zahn, R., Maglica, Z., Lee, S., Zentgraf, H., Weber-Ban, E. U., Dougan, D. A., Tsai, F. T., Mogk, A., and Bukau, B. (2004) Thermotolerance requires refolding of aggregated proteins by substrate translocation through the central pore of ClpB. Cell 119 (5), 653-665.

(12) Lee, S., Sowa, M. E., Watanabe, Y. H., Sigler, P. B., Chiu, W., Yoshida, M., and Tsai, F. T. (2003) The structure of ClpB: a molecular chaperone that rescues proteins from an aggregated state. Cell 115 (2), 229-240.

(13) Mazal, H., Iljina, M., Barak, Y., Elad, N., Rosenzweig, R., Goloubinoff, P., Riven, I., and Haran, G. (2019) Tunable microsecond dynamics of an allosteric switch regulate the activity of a AAA+ disaggregation machine. Nat. Commun. 10 (1), 1438.

(14) Lo, J. H., Baker, T. A., and Sauer, R. T. (2001) Characterization of the N-terminal repeat domain of Escherichia coli ClpA-A class I Clp/HSP100 ATPase. Protein Sci. 10 (3), 551-559.

(15) Wang, P., Li, J., Weaver, C., Lucius, A., and Sha, B. (2017) Crystal structures of Hsp104 N-terminal domains from Saccharomyces cerevisiae and Candida albicans suggest the mechanism for the function of Hsp104 in dissolving prions. Acta Crystallogr. D Struct Biol. 73 (4), 365-372.

(16) Squires, C. L., Pedersen, S., Ross, B. M., and Squires, C. (1991) $\mathrm{ClpB}$ is the Escherichia coli heat shock protein F84.1. J. Bacteriol. 173 (14), 4254-4262.
(17) Park, S. K., Kim, K. I., Woo, K. M., Seol, J. H., Tanaka, K., Ichihara, A., Ha, D. B., and Chung, C. H. (1993) Site-directed mutagenesis of the dual translational initiation sites of the clpB gene of Escherichia coli and characterization of its gene products. J. Biol. Chem. 268 (27), 20170-20174.

(18) Clarke, A. K., and Eriksson, M. J. (2000) The truncated form of the bacterial heat shock protein $\mathrm{ClpB} / \mathrm{HSP} 100$ contributes to development of thermotolerance in the cyanobacterium Synechococcus sp. strain PCC 7942. J. Bacteriol. 182 (24), 7092-7096.

(19) Doyle, S. M., Hoskins, J. R., and Wickner, S. (2012) DnaK chaperone-dependent disaggregation by caseinolytic peptidase $\mathrm{B}$ (ClpB) mutants reveals functional overlap in the N-terminal domain and nucleotide-binding domain-1 pore tyrosine. J. Biol. Chem. 287 (34), 28470-28479.

(20) Rosenzweig, R., Farber, P., Velyvis, A., Rennella, E., Latham, M. P., and Kay, L. E. (2015) ClpB N-terminal domain plays a regulatory role in protein disaggregation. Proc. Natl. Acad. Sci. U. S. A. 112 (50), E6872-E6881.

(21) Lee, S., Choi, J. M., and Tsai, F. T. (2007) Visualizing the ATPase cycle in a protein disaggregating machine: structural basis for substrate binding by ClpB. Mol. Cell 25 (2), 261-271.

(22) Carroni, M., Kummer, E., Oguchi, Y., Wendler, P., Clare, D. K., Sinning, I., Kopp, J., Mogk, A., Bukau, B., and Saibil, H. R. (2014) Head-to-tail interactions of the coiled-coil domains regulate $\mathrm{ClpB}$ activity and cooperation with Hsp70 in protein disaggregation. eLife 3, No. e02481.

(23) Yu, H., Lupoli, T. J., Kovach, A., Meng, X., Zhao, G., Nathan, C. F., and Li, H. (2018) ATP hydrolysis-coupled peptide translocation mechanism of Mycobacterium tuberculosis ClpB. Proc. Natl. Acad. Sci. U. S. A. 115 (41), E9560-E9569.

(24) Deville, C., Franke, K., Mogk, A., Bukau, B., and Saibil, H. R. (2019) Two-Step Activation Mechanism of the ClpB Disaggregase for Sequential Substrate Threading by the Main ATPase Motor. Cell Rep. 27 (12), 3433-3446 e4.

(25) Deville, C., Carroni, M., Franke, K. B., Topf, M., Bukau, B., Mogk, A., and Saibil, H. R. (2017) Structural pathway of regulated substrate transfer and threading through an Hsp100 disaggregase. Sci. Adv. 3 (8), No. e1701726.

(26) Rizo, A. N., Lin, J., Gates, S. N., Tse, E., Bart, S. M., Castellano, L. M., DiMaio, F., Shorter, J., and Southworth, D. R. (2019) Structural basis for substrate gripping and translocation by the ClpB AAA+ disaggregase. Nat. Commun. 10 (1), 2393.

(27) Gates, S. N., Yokom, A. L., Lin, J., Jackrel, M. E., Rizo, A. N., Kendsersky, N. M., Buell, C. E., Sweeny, E. A., Mack, K. L., Chuang, E., Torrente, M. P., Su, M., Shorter, J., and Southworth, D. R. (2017) Ratchet-like polypeptide translocation mechanism of the AAA+ disaggregase Hsp104. Science 357 (6348), 273-279.

(28) Barnett, M. E., Nagy, M., Kedzierska, S., and Zolkiewski, M. (2005) The amino-terminal domain of ClpB supports binding to strongly aggregated proteins. J. Biol. Chem. 280 (41), 34940-34945. (29) Mizuno, S., Nakazaki, Y., Yoshida, M., and Watanabe, Y. H. (2012) Orientation of the amino-terminal domain of ClpB affects the disaggregation of the protein. FEBS J. 279 (8), 1474-1484.

(30) Diemand, A. V., and Lupas, A. N. (2006) Modeling AAA+ ring complexes from monomeric structures. J. Struct. Biol. 156 (1), 230243.

(31) Barnett, M. E., Zolkiewska, A., and Zolkiewski, M. (2000) Structure and activity of $\mathrm{ClpB}$ from Escherichia coli. Role of the amino-and -carboxyl-terminal domains. J. Biol. Chem. 275 (48), 37565-37571.

(32) Beinker, P., Schlee, S., Groemping, Y., Seidel, R., and Reinstein, J. (2002) The $\mathrm{N}$ terminus of $\mathrm{ClpB}$ from Thermus thermophilus is not essential for the chaperone activity. J. Biol. Chem. 277 (49), 4716047166 .

(33) Nagy, M., Guenther, I., Akoyev, V., Barnett, M. E., Zavodszky, M. I., Kedzierska-Mieszkowska, S., and Zolkiewski, M. (2010) Synergistic cooperation between two $\mathrm{ClpB}$ isoforms in aggregate reactivation. J. Mol. Biol. 396 (3), 697-707. 
(34) Mogk, A., Schlieker, C., Strub, C., Rist, W., Weibezahn, J., and Bukau, B. (2003) Roles of individual domains and conserved motifs of the AAA+ chaperone $\mathrm{ClpB}$ in oligomerization, ATP hydrolysis, and chaperone activity. J. Biol. Chem. 278 (20), 17615-17624.

(35) Aguado, A., Fernández-Higuero, J. A., Cabrera, Y., Moro, F., and Muga, A. (2015) ClpB dynamics is driven by its ATPase cycle and regulated by the DnaK system and substrate proteins. Biochem. J. 466 (3), 561-570.

(36) Tripathi, P., Parijat, P., Patel, V. K., and Batra, J. K. (2018) The amino-terminal domain of Mycobacterium tuberculosis ClpB protein plays a crucial role in its substrate disaggregation activity. FEBS Open Bio 8 (10), 1669-1690.

(37) Sweeny, E. A., Tariq, A., Gurpinar, E., Go, M. S., Sochor, M. A., Kan, Z. Y., Mayne, L., Englander, S. W., and Shorter, J. (2020) Structural and mechanistic insights into $\mathrm{Hsp} 104$ function revealed by synchrotron x-ray footprinting. J. Biol. Chem. 295 (6), 1517-1538.

(38) Avellaneda, M. J., Franke, K. B., Sunderlikova, V., Bukau, B., Mogk, A., and Tans, S. J. (2020) Processive extrusion of polypeptide loops by a Hsp100 disaggregase. Nature 578 (7794), 317-320.

(39) Uchihashi, T., Watanabe, Y. H., Nakazaki, Y., Yamasaki, T., Watanabe, H., Maruno, T., Ishii, K., Uchiyama, S., Song, C., Murata, K., Ino, R., and Ando, T. (2018) Dynamic structural states of ClpB involved in its disaggregation function. Nat. Commun. 9 (1), 2147.

(40) Schütz, A. K., Rennella, E., and Kay, L. E. (2017) Exploiting conformational plasticity in the AAA+ protein $\mathrm{VCP} / \mathrm{p} 97$ to modify function. Proc. Natl. Acad. Sci. U. S. A. 114 (33), E6822-E6829.

(41) Pirchi, M., Tsukanov, R., Khamis, R., Tomov, T. E., Berger, Y., Khara, D. C., Volkov, H., Haran, G., and Nir, E. (2016) Photon-byPhoton Hidden Markov Model Analysis for Microsecond SingleMolecule FRET Kinetics. J. Phys. Chem. B 120 (51), 13065-13075.

(42) Woo, K. M., Kim, K. I., Goldberg, A. L., Ha, D. B., and Chung, C. H. (1992) The heat-shock protein ClpB in Escherichia coli is a protein-activated ATPase. J. Biol. Chem. 267 (28), 20429-20434.

(43) Kapusta, P., Wahl, M., Benda, A., Hof, M., and Enderlein, J. (2006) Fluorescence lifetime correlation spectroscopy. J. Fluoresc. 17 (1), 43-48.

(44) Aviram, H. Y., Pirchi, M., Mazal, H., Barak, Y., Riven, I., and Haran, G. (2018) Direct observation of ultrafast large-scale dynamics of an enzyme under turnover conditions. Proc. Natl. Acad. Sci. U. S. A. 115 (13), 3243-3248.

(45) Schlee, S., Groemping, Y., Herde, P., Seidel, R., and Reinstein, J. (2001) The chaperone function of ClpB from Thermus thermophilus depends on allosteric interactions of its two ATP-binding sites. J. Mol. Biol. 306 (4), 889-899.

(46) Diamant, S., Ben-Zvi, A. P., Bukau, B., and Goloubinoff, P. (2000) Size-dependent disaggregation of stable protein aggregates by the DnaK chaperone machinery. J. Biol. Chem. 275 (28), 2110721113.

(47) Tek, V., and Zolkiewski, M. (2002) Stability and interactions of the amino-terminal domain of $\mathrm{ClpB}$ from Escherichia coli. Protein Sci. 11 (5), 1192-1198.

(48) Marme, N., Knemeyer, J. P., Sauer, M., and Wolfrum, J. (2003) Inter- and intramolecular fluorescence quenching of organic dyes by tryptophan. Bioconjugate Chem. 14 (6), 1133-1139.

(49) Rosenzweig, R., Moradi, S., Zarrine-Afsar, A., Glover, J. R., and Kay, L. E. (2013) Unraveling the mechanism of protein disaggregation through a ClpB-DnaK interaction. Science 339 (6123), 1080-1083.

(50) Sharma, A., Enderlein, J., and Kumbhakar, M. (2017) Photon Antibunching Reveals Static and Dynamic Quenching Interaction of Tryptophan with Atto-655. J. Phys. Chem. Lett. 8 (23), 5821-5826.

(51) Oguchi, Y., Kummer, E., Seyffer, F., Berynskyy, M., Anstett, B., Zahn, R., Wade, R. C., Mogk, A., and Bukau, B. (2012) A tightly regulated molecular toggle controls AAA+ disaggregase. Nat. Struct. Mol. Biol. 19 (12), 1338-1346.

(52) Lipińska, N., Ziętkiewicz, S., Sobczak, A., Jurczyk, A., Potocki, W., Morawiec, E., Wawrzycka, A., Gumowski, K., Slusarz, M., Rodziewicz-Motowidło, S., Chruściel, E., and Liberek, K. (2013) Disruption of ionic interactions between the nucleotide binding domain 1 (NBD1) and middle (M) domain in Hsp100 disaggregase unleashes toxic hyperactivity and partial independence from Hsp70. J. Biol. Chem. 288 (4), 2857-2869.

(53) Kummer, E., Szlachcic, A., Franke, K. B., Ungelenk, S., Bukau, B., and Mogk, A. (2016) Bacterial and Yeast AAA+ Disaggregases ClpB and Hsp104 Operate through Conserved Mechanism Involving Cooperation with Hsp70. J. Mol. Biol. 428 (21), 4378-4391.

(54) Hayashi, S., Nakazaki, Y., Kagii, K., Imamura, H., and Watanabe, Y. H. (2017) Fusion protein analysis reveals the precise regulation between $\mathrm{Hsp} 70$ and $\mathrm{Hsp} 100$ during protein disaggregation. Sci. Rep. 7 (1), 8648.

(55) Sugita, S., Watanabe, K., Hashimoto, K., Niwa, T., Uemura, E., Taguchi, H., and Watanabe, Y. H. (2018) Electrostatic interactions between middle domain motif- 1 and the AAAl module of the bacterial $\mathrm{ClpB}$ chaperone are essential for protein disaggregation. $J$. Biol. Chem. 293 (50), 19228-19239.

(56) Watanabe, Y. H., Motohashi, K., and Yoshida, M. (2002) Roles of the two ATP binding sites of ClpB from Thermus thermophilus. J. Biol. Chem. 277 (8), 5804-5809.

(57) Seyffer, F., Kummer, E., Oguchi, Y., Winkler, J., Kumar, M., Zahn, R., Sourjik, V., Bukau, B., and Mogk, A. (2012) Hsp70 proteins bind Hsp100 regulatory $\mathrm{M}$ domains to activate AAA+ disaggregase at aggregate surfaces. Nat. Struct. Mol. Biol. 19 (12), 1347-1355.

(58) Durie, C. L., Duran, E. C., and Lucius, A. L. (2018) Escherichia coli DnaK Allosterically Modulates ClpB between High- and LowPeptide Affinity States. Biochemistry 57 (26), 3665-3675.

(59) Sweeny, E. A., Jackrel, M. E., Go, M. S., Sochor, M. A., Razzo, B. M., DeSantis, M. E., Gupta, K., and Shorter, J. (2015) The Hsp104 $\mathrm{N}$-terminal domain enables disaggregase plasticity and potentiation. Mol. Cell 57 (5), 836-849.

(60) Banerjee, S., Bartesaghi, A., Merk, A., Rao, P., Bulfer, S. L., Yan, Y., Green, N., Mroczkowski, B., Neitz, R. J., Wipf, P., Falconieri, V., Deshaies, R. J., Milne, J. L., Huryn, D., Arkin, M., and Subramaniam, S. (2016) $2.3 \AA$ resolution cryo-EM structure of human p97 and mechanism of allosteric inhibition. Science 351 (6275), 871-875.

(61) Nørby, J. G. (1988) Coupled assay of Na+,K+-ATPase activity. Methods Enzymol. 156, 116-119.

(62) Shorter, J. (2011) The mammalian disaggregase machinery: Hsp110 synergizes with $\mathrm{Hsp} 70$ and $\mathrm{Hsp} 40$ to catalyze protein disaggregation and reactivation in a cell-free system. PLoS One 6 (10), No. e26319.

(63) Mazal, H., Aviram, H., Riven, I., and Haran, G. (2018) Effect of ligand binding on a protein with a complex folding landscape. Phys. Chem. Chem. Phys. 20 (5), 3054-3062.

(64) Kapanidis, A. N., Lee, N. K., Laurence, T. A., Doose, S., Margeat, E., and Weiss, S. (2004) Fluorescence-aided molecule sorting: analysis of structure and interactions by alternating-laser excitation of single molecules. Proc. Natl. Acad. Sci. U. S. A. 101 (24), 8936-8941. 